\title{
Nouvelle méthode de maintenance CBM pour améliorer la durée de vie des pales d'éoliennes implantées dans les zones arides
}

\author{
Ahmida Rezig ${ }^{1}$, Ahmed Yousfi ${ }^{1}{ }^{*}$, Sylvain Fréour $^{2}$, Frédéric Jacquemin ${ }^{2}$ and Samuel \\ Branchu². \\ ${ }^{1}$ Mechanical Laboratory, University Amar Telidji of Laghouat, Algeria. \\ ${ }^{2} \mathrm{GeM}$ - Institut de Recherche en Génie Civil et Mécanique, UMR CNRS 6183, Université de \\ Nantes-École Centrale de Nantes, 58 rue Michel Ange, BP 420, 44606 Saint-Nazaire, France.
}

\begin{abstract}
Résumé: l'amélioration de la durée de vie des pales éoliennes opérant dans une région aride au-delà de sa durée de vie habituelle de 20 ou 25 ans oblige leur propriétaire à augmenter le nombre des inspections dans le cadre de la maintenance préventive sachant que les coûts de maintenance et d'arrêt d'une d'éolienne ont un impact direct sur la production totale et peuvent représenter jusqu'à $25 \%$ de l'énergie produite. Sachant que dans une région poussiéreuse, les pales sont soumises à une usure érosive qui contribue à la modification de la forme aérodynamique du bord d'attaque et réduit ainsi les performances de l'éolienne. Ce papier décrive et propose une nouvelle méthode de maintenance CBM pour améliorer la durée de vie d'une pale éolienne. Les résultats expérimentaux montrent que l'utilisation de cette méthode est très efficace pour détecter toute dégradation provoquée par l'usure érosive à la surface d'une pale pendant son service.
\end{abstract}

\section{Introduction}

Les composites sont souvent utilisés pour des pièces d'ingénierie dans les applications de l'aérospatiale, de l'automobile, de la marine et des pales d'éoliennes. En raison des exigences opérationnelles dans les environnements poussiéreux rencontrés dans les zones arides et semi-arides, les pales sont souvent exposées à l'érosion par les particules de sable. L'érosion des pales peut altérer leur géométrie, entraînant une perte de performance aérodynamique. L'érosion du matériau par des particules solides est un phénomène dont le processus est très complexe [2]. Les chocs entre les particules solides et la surface de la matière conduisent à l'enlèvement du matériau en raison d'une fracture fragile. Les types et dimensions des fissures qui se forment en surface lors de l'impact ont été étudiés de manière approfondie et dépendent de plusieurs facteurs: forme, masse, vitesse des particules, ductilité ou fragilité du matériau [3] and [4]. Les surfaces endommagées par l'érosion impliquent un énorme risque structurel, ce qui entraîne des temps d'immobilisation de la turbine et des coûts d'entretien élevés. En outre, une réparation tardive des sites érodés sur le bord d'attaque pour la restauration de sa forme aérodynamique d'origine peut

\footnotetext{
*Corresponding author: a.yousfi@lagh-univ.dz
} 
entraîner une fissuration puis une rupture de la pale. La dégradation du bord d'attaque de la pale résultant de l'érosion du sable entraîne une réduction considérable de ses performances. La rugosité de la pale à une grande influence sur les performances de l'éolienne et la production peut être réduite de 25\% [5]. Le besoin d'améliorer la précision de l'inspection préventive des défauts sur les bords d'attaque des pales d'éolienne conduit à installer un système de surveillance pour minimiser les incertitudes liées à l'inspection systématique des pales. Un système de surveillance permettra de réduire les coûts et le temps d'inspection ainsi que d'assurer la disponibilité de l'éolienne. Puisqu'un système de surveillance à distance est préférable à une inspection systématique, la réduction de l'envoi d'équipes de maintenance sera remarquable et les coûts devraient diminuer et la sécurité des intervenants s'améliorer. La tendance actuelle dans le secteur de l'énergie éolienne est d'établir un système de surveillance capable de détecter et de prévoir avec précision les pannes de pale sur les éoliennes de classe Megawatt.

Le but de ce travail est d'étudier la faisabilité d'un système de surveillance qui sera une partie intégrante de la maintenance conditionnelle sans inspection systématique et qui aura la capacité de détecter le niveau d'érosion en imposant un seuil bien défini. La méthodologie proposée dans cette étude est basée sur une conception structurelle utilisant une approche pour surveiller l'état du niveau d'érosion dans la couche externe d'une pale d'éolienne.

\subsection{L'érosion en fonction du temps de sablage}

Parmi plusieurs théories qui tentent de donner un modèle du volume $\Delta \mathrm{V}$ enlevé par l'érosion en fonction du temps d'exposition à l'impact des particules de sable $\Delta \mathrm{V}=f(\mathrm{t})$, celles suggérées par Barwell sont intéressantes [5]. Cette dernière est basée sur des équations empiriques directement issues des données expérimentales, cette théorie permette de prédire une moyenne du volume d'usure $\Delta \mathrm{V}$ subie par le matériau.

\section{Méthodes expérimentales}

L'appareil de fatigue par érosion est montré dans la figure 1 et qui comporte une chambre circulaire contenant du sable de silice avec une taille de particule variant de 40 à $80 \mu \mathrm{m}$. Elle est capable de créer un environnement presque identique à celui où les pales fonctionnent réellement. Pour effectuer les tests d'érosion, des échantillons de 50x50x1,371 $\mathrm{mm} 3$ ont été réalisés et montés sur un porte-échantillon spécialement conçu pour assurer des positions avec les angles de contact suivants $\left(\alpha: 20^{\circ}, 30^{\circ}, 45^{\circ}, 60^{\circ}, 75^{\circ}\right.$ et $\left.90^{\circ}\right)$. La durée totale du test de fatigue par usure érosive est de 458 heures, ce qui équivaut à 18 jours et 19 heures. Cette durée représente la durée totale du vent de sable pour un an selon les données météorologiques de la région de Laghouat - Algérie. Lorsque la couche externe est éliminée par l'érosion due à deux mécanismes distincts, à savoir l'usure par déformation et l'usure par découpe, le film d'isolation disparaît également permettant le passage des rayons UV des deux sources lumineuses installées sur la paroi de la chambre vers le film photovoltaïque. L'exposition du film PV aux rayons UV génère un courant électrique qui alimentera un circuit d'alarme électronique. Ce système d'alarme installé et protégé dans un coin du corps du ventilateur est équipé d'un émetteur qui émet un signal d'alarme à l'extérieur de la chambre et est capté par un autre circuit électronique (à environ $100 \mathrm{~m}$ de distance) déclenchant un signal sonore pour alerter que le seuil d'usure érosive est atteint. Lorsque le film isolant est enlevé, la couche de composite restante aura une épaisseur finale $e_{f}$ (couche seuil). Cette couche permettra le passage des rayons UV vers le film photovoltaïque générant un courant électrique dont l'intensité est proportionnelle à la valeur 
de $e_{f}$. Maintenant, si les particules de sable arrachent toutes les fibres, nous aurions atteint une épaisseur $\mathrm{e}_{\mathrm{T}}=0,6 \mathrm{~mm}$ (c'est-à-dire $\mathrm{Us}=0,8 \mathrm{~V}$ ), ceci représente l'épaisseur restante avant que l'érosion n'atteigne le film PV. La valeur de l'épaisseur restante sera égal à la valeur seuil de $\mathrm{e}_{\mathrm{f}}=0,6 \mathrm{~mm}$, après un temps d'exposition $\mathrm{T}=125 \mathrm{~h}$. La cellule photovoltaïque générera alors une tension $\mathrm{U}_{\mathrm{PV}}=0,8 \mathrm{~V}$. Lorsque le circuit électronique reçoit cette basse tension ( $\mathrm{Upv}=0,8 \mathrm{~V})$ il alimentera un commutateur électronique (IRBT); en conséquence, le circuit de l'émetteur se fermera et un signal sera envoyé à un autre circuit électronique situé à l'extérieur de l'appareil provoquant le déclenchement de l'alarme.

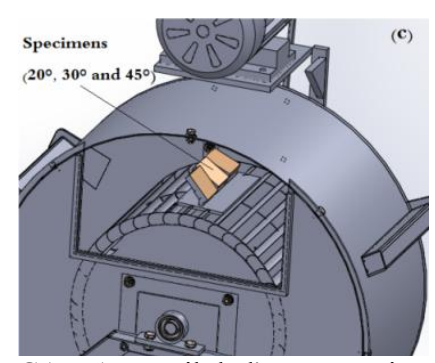

Fig.1: CAD Appareil de l'usure érosive.

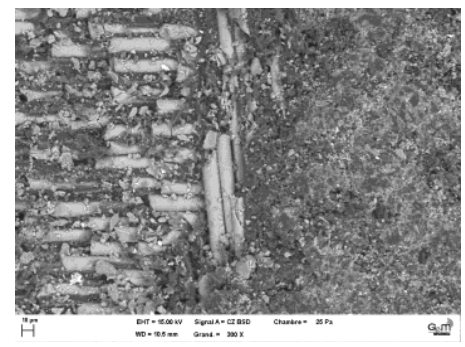

Fig.2: Image MEB montrant l'apparition des fibres après un temps d'exposition $\mathrm{T}=35 \mathrm{~h}$.

On peut voir sur la figure 2 que l'empilement des fibres de verre apparaît après un retrait intense du matériau de la matrice et est ensuite exposé à l'impact direct des particules de sable. Selon le système de surveillance, et sachant que l'épaisseur initiale, $\mathrm{e}_{\mathrm{i}}=1,371 \mathrm{~mm}$, l'épaisseur restante après un temps de test de $\mathrm{T} \approx 35 \mathrm{~h}$ est de $\mathrm{e}_{\mathrm{T}}=1,285 \mathrm{~mm}$ (c'est-à-dire à ce moment, Us $\approx 0,2 \mathrm{~V}$ ).

\section{Conclusion}

Le résultat expérimental recueilli a permis de conclure que la fatigue superficielle comme mécanisme de dégradation par usure peut expliquer l'intense érosion et la perte de matière observées des spécimens. Nous avons reproduit les effets des vents de sable en utilisant un appareil de fatigue érosive spécialement conçu. La surveillance de l'érosion du bord d'attaque des pales a permis de définir une maintenance CBM qui pourrait également s'appliquer sur des installations réelles afin d'intervenir juste à temps pour une réparation facile et rapide permettant de réduire les coûts d'exploitation. Un CBM bien établi peut réduire les coûts et les inspections de moitié. Un processus spécial en cours de fabrication nécessite un film PV sensible et son insertion entre les couches doit être optimisée pour couvrir les zones sensibles à l'érosion, c'est-à-dire les surfaces présentant des angles compris entre $50^{\circ}$ et $60^{\circ}$ par rapport la direction du vent.

\section{Références}

1. H.C. Meng, K.C. Ludema, Wear models and predictive equations: their form and content, Wear, 181-183 (1995), pp. 443-457

2. George F.SchmittJr, Solid particle erosion of reinforced composite materials, Wear, Volume 71, Issue 2, 8 September 1981, pp. 179-190

3. Iain Finnie, Erosion of surfaces by solid particles, Wear Volume 3, Issue 2, MarchApril 1960, pp. 87-103.

4. Sareen A, Chinmay S A, Selig M S, Effects of leading edge erosion on wind turbine blade performance, Wind Energy. Volume 17, issue10, 2014; pp.1531-1542.

5. F.T. Barwell, Wear of metals, Wear (1), 1957-1958, pp. 317-332. 